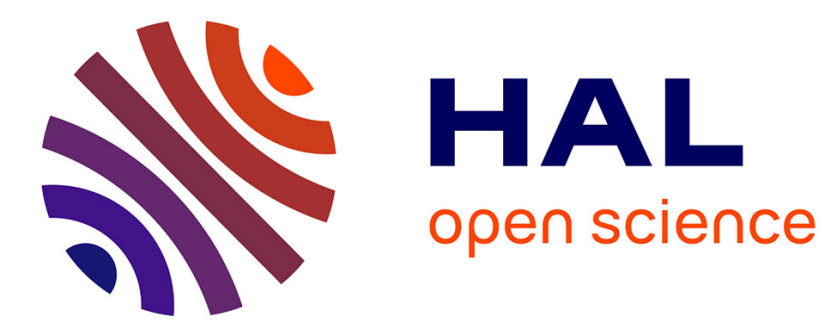

\title{
Approximate regulation for nonlinear systems in presence of periodic disturbances
}

Daniele Astolfi, Laurent Praly, Lorenzo Marconi

\section{To cite this version:}

Daniele Astolfi, Laurent Praly, Lorenzo Marconi. Approximate regulation for nonlinear systems in presence of periodic disturbances. 2015 54th IEEE Conference on Decision and Control (CDC), Dec 2015, Osaka, France. pp.7665-7670, 10.1109/CDC.2015.7403431 . hal-02297781

\section{HAL Id: hal-02297781 \\ https://hal.science/hal-02297781}

Submitted on 26 Sep 2019

HAL is a multi-disciplinary open access archive for the deposit and dissemination of scientific research documents, whether they are published or not. The documents may come from teaching and research institutions in France or abroad, or from public or private research centers.
L'archive ouverte pluridisciplinaire HAL, est destinée au dépôt et à la diffusion de documents scientifiques de niveau recherche, publiés ou non, émanant des établissements d'enseignement et de recherche français ou étrangers, des laboratoires publics ou privés. 


\title{
Approximate Regulation for Nonlinear Systems in Presence of Periodic Disturbances
}

\author{
Daniele Astolfi, Laurent Praly and Lorenzo Marconi
}

\begin{abstract}
In this work we present a new approach to the problem of output regulation for nonlinear systems in presence of periodic disturbances, possibly with an infinite number of harmonics. We show that, by adding a linear internal model, approximate regulation is achieved if the disturbance is small enough. Nominally all the harmonic included in the internal model are absent in the periodic steady state regulation error. Furthermore we show that the regulation error can be made arbitrarily small (in the $\mathcal{L}_{2}$ sense) by enlarging the dimension of the internal model. The novel approach relies on forwarding technique. An example is provided to show the efficacy of the result.
\end{abstract}

Index Terms-Output Regulation, Internal Model, Approximate Regulation, Nonlinear Systems.

\section{INTRODUCTION}

The problem of regulating desired outputs to some references while rejecting other external signals is generically known as a regulation problem. Regulation in the linear (multi-input multi-output) framework has been completely solved by Francis and Wonham (see [7]) during the 70's. In this contribution the authors made also clear what is the internal model principle, i.e. the fact that output regulation property is insensitive to plant parameter variations "only if the controller utilizes feedback of the regulated variable, and incorporates in the feedback path a suitably reduplicated model of the dynamic structure of the exogenous signals which the regulator is required to process".

Regulation in the nonlinear case, however, is somehow still an open problem due to the difficulties of extending the linear paradigm to a more general framework. Equivalent formulation of the regulation problem and the internal model principle in the nonlinear case has been developed during the 80's and especially in the 90's by many authors (see for instance [10] among the others). A breakthrough in the direction of solving the problem of output regulation for uncertain nonlinear systems was the crucial observation made in [13] (and independently in [8], [5] and [6]) that internal models must not only be able to generate inputs corresponding to the trajectories of the system, but also a number of higher order nonlinear deformations. In [2] a survey on the problem is given, where necessary conditions for the solvability of the problem and sufficient conditions for the problem of local regulation has been studied.

D. Astolfi is with CASY - DEI, University of Bologna, Italy and with MINES ParisTech, PSL Research University, CAS, Paris, France, (daniele.astolfi@unibo.it). L. Praly is with MINES ParisTech, PSL Research University, CAS, Paris, France, (laurent.praly@mines-paristech.fr). L. Marconi is with the CASY - DEI, University of Bologna, Italy, (lorenzo.marconi@unibo.it). This research has been partially framed within the collaborative project SHERPA (ICT 600958) supported by the European Community under the 7th Framework Program.
To overcome the problems raised by the nonlinearities, many different assumptions have been proposed in order to give a constructive solution to the regulation problem. Most of the recent works about regulation for nonlinear systems are using normal forms and minimum-phase assumptions. Essentially two different strategies are adopted in order to design the internal model. One approach relies on the immersion assumption (see [3]) by which the ideal steady state input can be written as a recursive function involving the derivatives up to a certain order. This assumption leads to write the internal model structure in the canonical observability form. A high-gain approach is then used to achieve exact regulation of the closed-loop system. Some recent works ([11], [16]) try to overcome the problem of nonminimum phase by applying the so called auxiliary problem first introduced in [9].

In [14] a different approach is proposed. The design of the internal model is made by the use of a Luenberger observer of appropriate dimension which asymptotically provides the so called "friend" (i.e. the ideal steady state input). It is worth noting that both the approaches rely on high-gain techniques, whereas in the linear framework high-gain tools are not needed. Among the different attempts that have been done in nonlinear output regulation, it is worth also recalling the approach in [4]. Therein the author proposes a design based on a high-gain observer, driven by the measured variables, which provides an estimate of the exogenous signal.

All the approaches proposed in the aforementioned works are focused on single input-single error systems. As shown in [1], the possible extension of those approaches to a general multi input-multi error framework is limited to a very specific class of square and invertible multivariable systems and hardly represents a good starting point to address, in its full generality, the multivariable framework. The main reason is that the proposed approaches follow a design paradigm of the form "add the internal model on the inputs and stabilize the resulting extended system", an approach not suitable in many multivariable contexts, such as the ones in which there are more inputs than measured variables. The different perspective proposed in [12], rather, follows the different paradigm "add the internal model on the errors and stabilize the resulting extended system", which is the perspective that is successfully used in the multivariable linear context ([7]). This motivated the attempt in [12], which addressed the problem of "shifting" the internal model from the input to the error for the specific class of single-input single-error nonlinear minimum-phases systems having a well-defined relative degree, and successfully extended to a particular class of multi-input multi-output nonlinear systems in [1]. 
In the same works the difficulties of pursuing the same approach to a more general class of nonlinear systems are also discussed.

In this work we present a new tool along the paradigm of "adding the internal model on the error and stabilizing the resulting extended system". We still insists on single inputsingle error systems but considering a very general class of nonlinear systems not necessarily minimum-phase and not necessarily possessing a relative degree. In particular, we develop the idea of approximate regulation, introduced for instance in [2]. Therein the authors considered the problem of output regulation for nonlinear systems driven by a linear exosystem. By augmenting the control law with a linear internal model of a number sufficiently high of harmonics, the authors show how to achieve local (in the initial conditions of the system and exosystem) approximate (up to a desired order) regulation. With respect to existing works, the approach pursued in this paper is new either for the synthesis of the control law and for the analysis of the results. The result can be seen as an extension of [17] in which the same design and analysis philosophy pursued here was followed in the specific case of constant disturbances. Here we extend that framework to the case in which the disturbance is periodic. The result is based on the well known fact that the solution of a nonlinear ISS (Input-to-State Stable) system, driven by a periodic input, is itself a periodic function of the same period. The analysis is driven by Fourier analysis. By embedding the internal model with a certain number of harmonics, we are then able to prove that the spectral components of the output with the same frequencies are not present in the output signal. Approximate regulation follows as an application of the Parseval's identity.

Notation. $\mathrm{i}$ is the imaginary unit (i.e. $\mathrm{i}=\sqrt{-1}$ ). $\lceil x\rceil$ is the smallest integer $n$ larger than $x$. Given a $T$-periodic signal $s(t)$, we define its $\mathcal{L}_{2}$ norm and its $\mathcal{L}_{\infty}$ norm as

$$
\|s(t)\|_{2}:=\sqrt{\frac{1}{T} \int_{0}^{T}|s(t)|^{2} d t}, \quad\|s(t)\|_{\infty}:=\max _{t \geq 0}|s(t)| .
$$

\section{The MOTIVATING LINEAR CONTEXT}

The motivating context for the approach to (approximate) nonlinear output regulation followed in this paper is given by the linear framework developed in its full generality in the milestone paper [7]. Consider the linear system

$$
\begin{aligned}
\dot{x} & =A x+B u+P w \\
e & =C_{e} x+Q_{e} w \\
y & =C_{y} x+Q_{y} w
\end{aligned}
$$

with state $x \in \mathbb{R}^{n}$, control input $u \in \mathbb{R}^{m}$, regulated measured output $e \in \mathbb{R}^{p}$ and extra set of measured outputs $y \in \mathbb{R}^{r}$. The exogenous variable $w$ is generated by the exosystem $\dot{w}=S w, w \in \mathbb{R}^{s}$, with $S$ assumed to be neutrally stable. We assume $(A, B)$ is controllable and the pair $\left(A,\left(C_{e}^{T}, C_{y}^{T}\right)^{T}\right)$ is observable, namely system (1) with $w=0$ is stabilizable by output feedback. The problem of output regulation amounts to designing a dynamic controller, processing the measures $(e, y)$, such that the resulting closedloop system has bounded trajectories and the regulated error $e$ is asymptotically vanishing. The solution to the problem leads to the celebrated internal model principle, claiming that the controller solving the problem necessarily embeds suitable copies of the exosystem. In rough words the recipe for designing the controller follows the following two steps:

1 add $p$ copies of the exosystem processing the errors, one for each error $e$, which represent the internal model of the exosystem;

2 stabilize the resulting cascade system with $w=0$ regarded as a system with input $u$, and measured outputs given by $(e, y)$ and by the state of the internal model.

In more precise terms, the internal model is a system of the form

$$
\dot{\xi}=\mathbf{\Phi} \xi+\mathbf{G} e
$$

with $\xi=\operatorname{col}\left(\xi_{1}, \ldots, \xi_{p}\right), \xi_{i} \in \mathbb{R}^{\nu}, i=1, \ldots, p$,

$$
\boldsymbol{\Phi}=\operatorname{blkdiag}(\underbrace{\phi, \ldots, \phi}_{p \text { times }}), G=\operatorname{blkdiag}(\underbrace{G, \ldots, G}_{p \text { times }}),
$$

where $(\phi, G)$ is a controllable pair with $\phi$ such that it's characteristic polynomial coincides with the minimal polynomial of $S$. The cascade system so obtained is then regarded as a system with input $u$ and output $y_{e}=(e, y, \xi)$. Such a system is always observable by the output $y_{e}$ (as a consequence of the observability of the regulated system (1) and of the fact that $\xi$ is measured). Furthermore, by applying the PBH test, such a cascade is controllable by the input $u$ if the following non-resonance condition is fulfilled

$$
\operatorname{rank}\left(\begin{array}{ccc}
A-\lambda I_{n} & 0 & B \\
G C_{e} & \mathbf{\Phi}-\lambda I_{\nu p} & 0
\end{array}\right)=n+\nu p .
$$

It is worth noting that the previous non-resonance condition necessarily asks that $m \geq p$, namely that the number of inputs is not lower than the number of regulated errors. Then, if the previous condition (3) is fulfilled, there exists a controller of the form

$$
\begin{aligned}
& \dot{\eta}=F \eta+L y_{e} \\
& u=M \eta+N y_{e}
\end{aligned}
$$

such that the closed-loop system given by (1), (2) and (4) with $w=0$ is Hurwitz. By partitioning the matrices $L$ and $N$ as $L=\left(L_{e}, L_{y}, L_{\xi}\right)$ and $N=\left(N_{e}, N_{y}, N_{\xi}\right)$ according to the definition of $y_{e}$, the resulting internal model-based controller is thus of the form

$$
\begin{aligned}
\left(\begin{array}{c}
\dot{\xi} \\
\dot{\eta}
\end{array}\right) & =\left(\begin{array}{cc}
\mathbf{\Phi} & 0 \\
L_{\xi} & F
\end{array}\right)\left(\begin{array}{l}
\xi \\
\eta
\end{array}\right)+\left(\begin{array}{cc}
\mathbf{G} & 0 \\
L_{e} & L_{y}
\end{array}\right)\left(\begin{array}{c}
e \\
y
\end{array}\right) \\
u & =\left(\begin{array}{ll}
N_{\xi} & M
\end{array}\right)\left(\begin{array}{l}
\xi \\
\eta
\end{array}\right)+\left(\begin{array}{ll}
N_{e} & N_{y}
\end{array}\right)\left(\begin{array}{l}
e \\
y
\end{array}\right)
\end{aligned}
$$

with the resulting closed-loop system that, denoting by $z=$ $\operatorname{col}(x, \xi, \eta)$ the full state, can be compactly written as

$$
\begin{aligned}
\dot{w} & =S w \\
\dot{z} & =A_{\mathrm{cl}} z+P_{\mathrm{cl}} w
\end{aligned}
$$

for appropriately defined matrices $A_{\mathrm{cl}}$ and $P_{\mathrm{cl}}$. Due to the fact that $S$ is neutrally stable and $A_{\mathrm{cl}}$ is Hurwitz, the closedloop trajectories are clearly bounded and, having defined by $\Pi_{z}$ the solution of the Sylvester equation

$$
\Pi_{z} S-A_{\mathrm{cl}} \Pi_{z}=P_{\mathrm{cl}},
$$


standard arguments can be used to show that the following holds

$$
\lim _{t \rightarrow \infty}\left(z(t)-\Pi_{z} w(t)\right)=0 .
$$

Namely, the closed loop trajectories reach a steady state given by $\Pi_{z} w(t)$. Furthermore, by partitioning $\Pi_{z}$ as $\Pi_{z}=$ $\operatorname{col}\left(\Pi_{x}, \Pi_{\xi}, \Pi_{\eta}\right)$ coherently with the definition of $z$, it turns out that the fact that the characteristic polynomial of $\phi$ coincides with the minimal polynomial of $S$ can be used to prove that

$$
C_{e} \Pi_{x}+Q_{e}=0,
$$

by which we conclude that the regulation error converges to zero asymptotically. Remarkably, the regulation requirements are fulfilled also in presence of changes in the dynamics (1), for instance due to uncertainties in the controlled plant, as long as the stabilizer (4) asymptotically stabilizes the extended system (property known as structurally stable regulation).

The solution for linear systems presented above, following the paradigm "add oscillators on the error and stabilize the extended system", inspires also the approach presented in the next section for nonlinear systems. In the nonlinear context, however, we cannot expect that the addition of a finite number of oscillators is enough to have zero regulation error, since an infinite number of harmonics is, in general, needed to have perfect regulation. Namely, nonlinear internal model are, in general, needed. The best one can expect is that the addition of oscillators leads to a reduction of the regulation error, namely that practical regulation is achieved with the error bound that decreases as new harmonics are included in the internal model. This, indeed, is the main conclusion drawn in the next section for the specific class of periodic exosystems and for a fairly general class of nonlinear systems.

\section{NONLINEAR OUTPUT REGULATION} form

We consider nonlinear systems affine in the input of the

$$
\dot{x}=f(x, d)+g(x, d) u, \quad e=h(x, d),
$$

with state $x \in \mathbb{R}^{n}$, control input $u \in \mathbb{R}$ and regulated output $e \in \mathbb{R}$. The mappings $f: \mathbb{R}^{n} \times \mathbb{R}^{n_{d}} \rightarrow \mathbb{R}^{n}, g: \mathbb{R}^{n} \times \mathbb{R}^{n_{d}} \rightarrow$ $\mathbb{R}^{n}, h: \mathbb{R}^{n} \times \mathbb{R}^{n_{d}} \rightarrow \mathbb{R}$ are assumed to be smooth enough. The exogenous signal $d(t)$, which can be considered as a disturbance to be rejected or a reference to be tracked, is assumed to be a continuous periodic signal of known period $T$. The exogenous signal could be thought of as generated by an exosystem of the form $\dot{w}=s(w), d=\gamma(w)$ even though the forthcoming analysis doesn't necessarily rely upon this description.

In order to streamline the presentation, in this paper we assume that the full state of the system and the regulated error $e$ are accessible for feedback. All the design procedure illustrated next, however, can be extended to the case in which only some measured outputs (plus the regulated error) are available provided that observability conditions are satisfied. In this respect the main assumption on the regulated plant is that the origin of (5) with $d=0$ is stabilizable by state feedback with a given region of attraction. Without loss of generality we thus assume that a stabilizer has been already designed for the system so that the origin of (5) with $d=0$ and $u=0$ is locally asymptotically stable. More formally we assume the following.

Assumption 1 The system $\dot{x}=f(x, 0)$ is asymptotically and locally exponentially stable with domain of attraction an open set $\mathcal{S} \subset \mathbb{R}^{n}$.

Following the paradigm illustrated in the previous section for linear systems, the system (5) is augmented with an integrator ${ }^{1}$ and a bunch of $L$ oscillators all driven by the error $e$, whose frequencies are multiple of the basic frequency characterizing the disturbance $d$, namely

$$
\dot{z}_{\ell}=-\mathrm{i} \ell \frac{2 \pi}{T} z_{\ell}+h(x, d), \quad \ell \in\{0,1, \ldots, L\},
$$

with $z_{\ell} \in \mathbb{C}$. The number of harmonics $L$ will be used next as a design parameter to enforce a small regulated error. Note that there exists a real realization of order $1+2 L$ of system (6). In the following we shall denote such a real representation as

$$
\dot{z}=\phi_{L} z+G h(x, d), \quad z \in \mathbb{R}^{1+2 L},
$$

where $\phi_{L}$ and $G$ are appropriately defined matrices.

Coherently with the linear analysis presented in the previous section, the first result that is given below regards the stabilisability of the extended system (5)-(6) when the exogenous disturbance is absent. The stabilisability of the cascade relies on a non-resonance condition that, in the actual nonlinear setting, is formulated as follow.

Assumption 2 The following condition holds

$$
\operatorname{rank}\left(\begin{array}{cc}
F-\lambda I & G \\
H & 0
\end{array}\right)=n+1 \quad \forall \lambda=\ell \frac{2 \pi}{T} \mathrm{i}
$$

for all $\ell=0,1, \ldots, L$, where

$$
F=\frac{\partial f}{\partial x}(0,0), \quad G=g(0,0), \quad H=\frac{\partial h}{\partial x}(0,0) .
$$

This assumption states that the first order approximation at the origin of the system has zeros that are not synchronous with the frequencies of the internal model. Under the previous assumptions there always exists a state feedback control law stabilizing the origin of (5)-(6) when the disturbance is not present. This is formulated in the next proposition that also claims that the amplitude of the stabilizing control law is arbitrary.

Proposition 1 Consider the cascade (5),(7) with $d(t) \equiv 0$ and $L \in \mathbb{N}$ fixed. Let Assumptions 1 and 2 hold. For any $\bar{u}>0$ there exists a bounded state feedback control law $\phi_{\bar{u}}: \mathbb{R}^{n} \times \mathbb{R}^{1+2 L} \rightarrow \mathbb{R}$, with $\left|\phi_{\bar{u}}(\cdot, \cdot)\right| \leq \bar{u}$, such that the origin of system (5), (7) in closed loop with $u=\phi_{\bar{u}}(x, z)$ is asymptotically stable and locally exponentially stable with $\mathcal{S} \times \mathbb{R}^{1+2 L}$ as domain of attraction.

\footnotetext{
${ }^{1}$ Note that in the linear case, if the exogenous signal $d(t)$ has zero mean value the integral action is not needed. This condition, in general, is not sufficient when nonlinearities are present. For instance the signal $d(t)=$ $\sin (t)$ has zero mean value, but $d(t)^{2}$ no.
} 
The proof of this proposition follows by using forwarding techniques noting that the cascade is in feedforward form (see for instances [15], [17]). Clearly the stabilizing control law $\phi_{\bar{u}}(\cdot)$ depends on $L$ that thus must be fixed beforehand. The previous proposition refers to the system with $d(t) \equiv$ 0 . When $d$ is not zero, by using standard total stability arguments ([18]), it is possible to show that if $d(t)$ is bounded, periodic and small enough, there exists a periodic asymptotically stable solution for the closed-loop extended system. This is formalized in the next proposition.

Proposition 2 Consider the cascade (5),(7) in closed-loop with a stabilizing feedback $u=\phi_{\bar{u}}(x, z)$. Then, for any compact set $C_{x} \times C_{z} \subset \mathbb{R}^{n} \times \mathbb{R}^{1+2 L}$, containing the origin and with $C_{x} \subset \mathcal{S}$, there exists a real number $\bar{d}_{1}>0$ such that, for all $t \mapsto d(t) T$-periodic satisfying $|d(t)| \leq \bar{d}_{1}$, there exists a T-periodic trajectory $\left(x^{\star}(t), z^{\star}(t)\right) \in C_{x} \times C_{z}$ which is asymptotically stable and locally exponentially stable with $\mathcal{X} \times \mathcal{Z}$ as domain of attraction, where $C_{x} \subset \mathcal{X} \subset \mathcal{S}$ and $C_{z} \subset \mathcal{Z} \subset \mathbb{R}^{1+2 L}$.

It is worth observing that the real number $\bar{d}_{1}>0$ depends on $\phi_{\bar{u}}(x, z)$. Motivated by the previous proposition we now study the properties of the cascade system along periodic trajectories. The main result in this direction is presented in the following proposition in which, for a periodic error function $e(t)$, we denote by $c_{k}^{0}$ the $k$-th Fourier coefficient of $e$, i.e.

$$
c_{k}^{0}=\frac{1}{T} \int_{0}^{T} e(t) \exp \left(2 \mathrm{i} k \pi \frac{t}{T}\right) d t
$$

Proposition 3 Let $(x(t), z(t))$ be a bounded trajectory of the cascade (5)-(7) such that $e(t+T)=e(t)$ for all $t \geq 0$. Then necessarily

$$
c_{k}^{0}=0 \quad \forall k=0,1, \ldots, L .
$$

Moreover, for any compact set $C_{x} \subset \mathbb{R}^{n}$, for any $\bar{d}_{1}>0$, $\bar{d}_{2}>0, \bar{u}>0$ and $\varepsilon>0$ such that $x(t) \in C_{x},|d(t)| \leq \bar{d}_{1}$, $|\dot{d}(t)| \leq \bar{d}_{2}$ and $|u(t)| \leq \bar{u}$ for all $t \geq 0$, there exists $L^{*} \geq 1$ such that

$$
\|e(t)\|_{2} \leq \varepsilon \quad \forall L \geq L^{*} .
$$

Proof: Consider the system (6). It is a a linear system driven by a periodic function $e(t)$ and the solution $z(t)$ is bounded. For any $\ell \in\{0,1, \ldots, L\}$ consider the solution $z_{\ell}(t)$ along a period $T$

$$
\begin{aligned}
z_{\ell}(t+T)=\exp (-\mathrm{i} \ell \omega T) z_{\ell}(t) & \\
& +\int_{t}^{T+t} \exp (-\mathrm{i} \ell \omega(T+t-s)) e(s) \mathrm{d} s,
\end{aligned}
$$

where we denoted $\omega=\frac{2 \pi}{T}$. Without loss of generality we pick $t=0$. As a consequence

$$
0=\int_{0}^{T} \exp (\mathrm{i} \ell \omega s) e(s) \mathrm{d} s,
$$

and by using definition (8) we get $c_{\ell}=0$ for any $\ell \in$ $\{0,1, \ldots, L\}$. This completes the first part of the proof.
Now let $H_{1}, F_{0}$ and $D_{1}$ be the real numbers defined as

$$
\begin{aligned}
H_{1} & =\sup _{x \in C_{x},|d| \leq \bar{d}_{1}}\left\{\frac{\partial h}{\partial x}(x, d)\right\}, \\
F_{0} & =\sup _{x \in C_{x},|d| \leq \bar{d}_{1},|u| \leq \bar{u}}\{f(x, d)+g(x, d) u\}, \\
D_{1} & =\sup _{x \in C_{x},|d| \leq \bar{d}_{1},|\dot{d}| \leq \bar{d}_{2}}\left\{\frac{\partial h}{\partial d}(x, d) \frac{\partial d}{\partial t}(t)\right\} .
\end{aligned}
$$

It follows that $|\dot{e}(t)| \leq H_{1} F_{0}+D_{1}$ for all $t \geq 0$. Moreover, along any solution, the function $t \mapsto(e(t), \dot{e}(t))$ is continuous and thus square integrable on $[0, T]$. It follows that $e(t)$ and $\dot{e}(t)$ can be expressed by a Fourier Series

$$
\begin{aligned}
& e(t)=\sum_{k=0}^{\infty} c_{k}^{0} \exp (\mathrm{i} k \omega t), \\
& \dot{e}(t)=\sum_{k=0}^{\infty} \mathrm{i} k \omega c_{k}^{0} \exp (\mathrm{i} k \omega t)=\sum_{k=0}^{\infty} c_{k}^{\prime} \exp (\mathrm{i} k \omega t),
\end{aligned}
$$

where $c_{k}^{\prime}=\mathrm{i} k \omega c_{k}^{0}$. By using Parseval's Identity we get

$$
\sqrt{\frac{1}{T} \int_{0}^{T}|\dot{e}(s)|^{2} \mathrm{~d} s}=\sqrt{2 \sum_{k=0}^{\infty}\left(c_{k}^{\prime}\right)^{2}} \leq H_{1} F_{0}+D_{1} .
$$

From the previous result we know that $c_{k}^{0}=0$ for all $\ell \in$ $\{0,1, \ldots L\}$. As a consequence (and by using the definition of $c_{k}^{\prime}$ ) we get

$\omega^{2}(L+1)^{2} \sum_{k=L+1}^{\infty}\left(c_{k}^{0}\right)^{2} \leq \sum_{k=L+1}^{\infty}\left(k \omega c_{k}^{0}\right)^{2} \leq \frac{\left(H_{1} F_{0}+D_{1}\right)^{2}}{2}$.

Again, by using Parseval's Identity, we get

$$
\frac{1}{T} \int_{0}^{T}|e(s)|^{2} \mathrm{~d} s \leq \frac{\left(H_{1} F_{0}+D_{1}\right)^{2}}{\omega^{2}(L+1)^{2}} .
$$

The proof completes by setting $L^{*}=\left[\frac{\left(H_{1} F_{0}+D_{1}\right) T}{2 \pi \varepsilon}\right]$.

It is worth noting that, when specialized to linear systems and to the case of disturbances $d(t)$ with a finite number of harmonics, the first part of the Proposition 3 along with the result of Propositions 1 and 2 leads to the conclusion that the regulator $u=\phi_{\bar{u}}(x, z)$, with $z$ generated by the internal model (7), solves the problem of asymptotic output regulation (namely the error converges to zero asymptotically) provided that all the harmonics characterizing $d(t)$ are included in $\phi_{L}$. As a matter of fact, in the linear case, the closed-loop solutions converge to the periodic steady state trajectory $\left(x^{\star}(t), y^{\star}(t)\right)$ which is a periodic function with the same harmonic content as $d(t)$. This implies that also the regulation error reaches a periodic steady state with the same harmonic content as $d(t)$. Moreover, since its Fourier coefficients associated to the harmonics that are contained in $\phi_{L}$ are necessarily zero by the previous proposition, asymptotic regulation is fulfilled. Clearly, in the linear case, the result is global, namely it holds for all initial conditions of the extended system and for all (arbitrarily large) periodic disturbances. In case of nonlinear systems or periodic disturbances with an infinite number of harmonics, the first part of the previous proposition just guarantees that the harmonics included in the internal model are absent in 
the steady state error, without any evident benefit in terms of regulation error. In this respect the second part of the proposition claims that the error can be arbitrarily decreased (in the $\mathcal{L}_{2}$ sense) by adding harmonic on the internal model, namely by increasing the value of $L$. By combining the results in the previous propositions it is then possible to claim the following theorem which is the main result of the paper.

Theorem 1 Let Assumptions 1 and 2 hold. Let $C_{x}$ be a compact set contained in $\mathcal{S}$, and $\bar{u}>0$ and $\bar{d}_{2}>0$. Then, for any $\varepsilon>0$ there exists $L^{\star}>0$ and, for any $L \geq L^{\star}$, there exist a $\phi_{\bar{u}}(x, z)$ and $\bar{d}_{1}>0$ such that the solution of system (5)-(7) in closed loop with $u=\phi_{\bar{u}}(x, z)$ with initial condition $(x(0), z(0)) \in C_{x} \times\{0\}$ and $t \mapsto d(t) T$-periodic with $|d(t)|_{\infty} \leq \bar{d}_{1},|\dot{d}(t)|_{\infty} \leq \bar{d}_{2}$, is bounded, T-periodic and such that $c_{k}^{0}=0$ for all $k \leq L$ and $\|e(t)\|_{2} \leq \varepsilon$.

\section{EXAMPLE}

We consider the following system

$$
\dot{\xi}=\varphi(\xi, u, d), \quad e=h(\xi, d)
$$

where $\xi \in \mathbb{R}^{2}, e \in \mathbb{R}, u \in \mathbb{R}$ and

$$
\varphi(\xi, u, 0)=\operatorname{col}\left(\xi_{1}^{3}+\xi_{2}, u\right), \quad h(\xi, d)=\xi_{2} .
$$

Before testing the design procedure presented in Section III, it is worth remarking a few properties of the system. First, the system with input $u$ and output $e$ is non-minimum phase. This implies that, to stabilize the system, the control input $u$ must necessarily embed a $\xi_{1}$ component whose steady state is not zero and, moreover, it must necessarily be equal to zero when output regulation is achieved. We also observe that the component $\xi_{1}$ is not observable from the output $e$, thus a state-feedback law is needed. Further, we do not specify how $\varphi$ depends on $d$ and we make no assumption on how $d$ is generated except that it is $C^{1}$ and periodic.In particular it may not satisfy an immersion assumption (see [3]), i.e. it may have spectral components at an infinite number of frequencies. Hence, we have here an example where most of the recent and common techniques which relies on a minimum-phase assumption [3], [14], on the notion of friend [14], or on an immersion assumption [3], [16], [4], cannot be applied.

For all non zero $\lambda$, we have

$$
\operatorname{rank}\left(\begin{array}{cc}
F-\lambda I & G \\
H & 0
\end{array}\right)=\operatorname{rank}\left(\begin{array}{cc|c}
-\lambda & 1 & 0 \\
0 & -\lambda & 1 \\
\hline 0 & 1 & 0
\end{array}\right)=3 .
$$

Hence Assumption 2 is satisfied except that we cannot add an integral action (i.e. $\ell$ cannot be zero). Concerning Assumption 1, by using standard backstepping techniques, it can be easily checked that by choosing $u=\phi_{1}(\xi)+v$ where

$$
\phi_{1}(\xi)=-\left(3 \xi_{1}^{2}+a_{1}\right)\left(\xi_{2}+\xi_{1}^{3}\right)-a_{2}\left(\xi_{1}^{3}+a_{1} \xi_{1}+\xi_{2}\right)
$$

with $a_{1}>0, a_{2}>0$, and by applying the change of coordinates

$$
\begin{aligned}
& \xi_{1} \mapsto x_{1}:=\xi_{1} \\
& \xi_{2} \mapsto x_{2}:=a_{1} \xi_{1}+\xi_{1}^{3}+\xi_{2}
\end{aligned}
$$

the system (9) appears as

$$
\dot{x}=A x+B v, \quad e=h(x),
$$

where, keeping the same symbol $h, h(x)=x_{2}-a_{1} x_{1}-x_{1}^{3}$ and

$$
A=\left(\begin{array}{cc}
-a_{1} & 1 \\
0 & -a_{2}
\end{array}\right), \quad B=\left(\begin{array}{l}
0 \\
1
\end{array}\right) .
$$

This establishes that Assumption 1 holds after a preliminary feedback. Following the previous section we extend (12) with the following internal model

$$
\dot{z}=\phi_{L} z+G h(x), \quad z \in \mathbb{R}^{2 L},
$$

where $L$ is a natural number to be fixed. Following standard forwarding techniques ([15]), a stabilizing law for the cascade system (12), (13) is $v=\phi_{2}(x, z)$ with

$\phi_{2}(x, z)=\left[\left(c_{2}\left(z-\mathcal{M}_{L}(x)\right)^{\top} \frac{\partial \mathcal{M}_{L}}{\partial x}(x)-c_{1} \frac{\partial V}{\partial x}(x)\right) B\right]_{(14)}^{\top}$

where $c_{1}>0, c_{2}>0, \mathcal{M}_{L}(x)$ is a matrix whose entries are polynomials of degree 3 in $x$, computed as solution of

$$
\frac{\partial \mathcal{M}_{L}}{\partial x}(x) A x=\phi_{L} \mathcal{M}_{L}(x)+G h(x)
$$

and $V(x)=\frac{1}{2}\left(x_{1}^{2}+x_{2}^{2}\right)$. This can be verified with the following Lyapunov function

$$
U(x, z)=V(x)+\left(z-\mathcal{M}_{L}(x)\right)^{\top}\left(z-\mathcal{M}_{L}(x)\right) .
$$

Proposition 1 is then satisfied by setting

$$
\begin{gathered}
\phi_{\bar{u}}(\xi, z)=\operatorname{sat}_{\bar{u}}\left[\phi_{1}(\xi)+\phi_{2}(x, z)\right], \\
\operatorname{sat}_{\bar{u}}(s)=\min \left\{1, \frac{\bar{u}}{|s|}\right\} s,
\end{gathered}
$$

with $x$ obtained from $\xi$ via (11) and where $\bar{u}>0$.

In the simulations $d(t) \leq 1$ is a periodic zero mean value signal obtained by filtering with a high-pass filter the signal $\varrho(t)$, generated as

$$
\begin{aligned}
\varrho(t)= & \sin (\sin (2 \pi t)+\sin (4 \pi t-0.2)+ \\
& \sin (6 \pi t+0.7)+\sin (10 \pi t+0.5)) .
\end{aligned}
$$

Figure 1 shows two periods of the signal $d(t)$. The basic frequency of $d(t)$ is $1 \mathrm{~Hz}$ but it has spectral components at many higher harmonics, as shown in Figure 2 (obtained by using the command fft in MATLAB). In the design of (15) the parameters have been taken as $a_{1}=5, a_{2}=3$, $c_{1}=1, c_{2}=50$ and $\bar{u}=50$. Figure 3 shows the behavior of the spectral components of the periodic solution $e(t)$ in the case there is no internal model (this is obtained by choosing $c_{2}=0$ in the control law (14)) and in the case where the internal model is present, with $L=1, \ldots, 5$. The magnitude of the disturbance is not changed in the different simulations. It can be seen from the figure that each time that an oscillator is added, the relative spectral component is eliminated, and consequently the $\mathcal{L}_{2}$ norm of the output $e(t)$ is reduced (as shown in Table 1).

TABLE I: $\mathcal{L}_{2}$ norm and $\mathcal{L}_{\infty}$ norm of the error.

\begin{tabular}{lll}
$L=0$ & $\|e(t)\|_{2}=2.0916$ & $\|e(t)\|_{\infty}=0.2351$ \\
$L=1$ & $\|e(t)\|_{2}=1.0823$ & $\|e(t)\|_{\infty}=0.0740$ \\
$L=2$ & $\|e(t)\|_{2}=0.7495$ & $\|e(t)\|_{\infty}=0.0392$ \\
$L=3$ & $\|e(t)\|_{2}=0.5806$ & $\|e(t)\|_{\infty}=0.0205$ \\
$L=4$ & $\|e(t)\|_{2}=0.4683$ & $\|e(t)\|_{\infty}=0.0125$ \\
$L=5$ & $\|e(t)\|_{2}=0.2428$ & $\|e(t)\|_{\infty}=0.0049$ \\
\hline
\end{tabular}




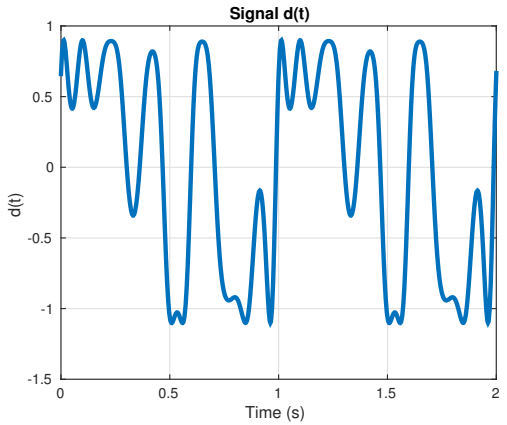

Fig. 1: Two periods of the disturbance $d(t)$.

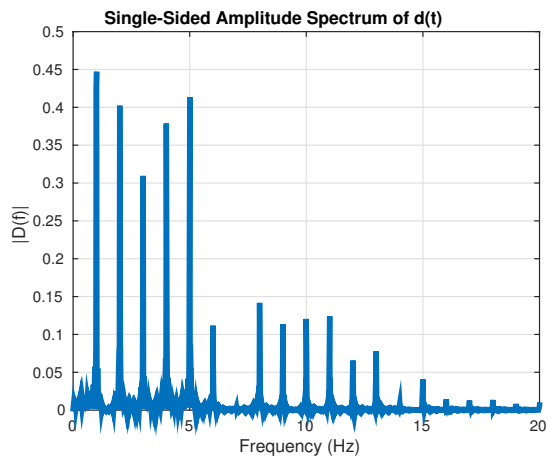

Fig. 2: Frequency spectrum of the disturbance $d(t)$.

\section{CONCLUSION}

In this work we studied the problem of approximate regulation for nonlinear systems in the case of periodic disturbances. We considered a class of systems which are affine in the inputs, possibly non-minimum phase, for which it may not exist a normal form or for which the relative degree may not be well-defined. A linear internal model processing the output is added. The state-feedback design relies on the forwarding technique and it provides asymptotic stability of the origin when the disturbance is not present. If the disturbance is not too large then practical regulation is achieved and we show that the harmonics included in the internal model are absent in the periodic steady state regulation error. Finally we show that the error can be arbitrarily decreased (in the $\mathcal{L}_{2}$ sense) by enlarging the dimension of the internal model. The proposed technique can be easily extended to a more general class of multi-input multi-output (possibly non-square) nonlinear systems.

Two main questions are still open and further work needs to be done in order to have a complete answer. First, in Theorem 1 , we do not know how the bound $\bar{d}_{1}$ on the disturbance depends on the number of oscillators $L$ in the internal model. The technical arguments we have found up to now seem to say, the larger $L$ is, the smaller $\bar{d}_{1}$ shloud be. But this is not confirmed by our simulations. Second, it is still unclear if we can get a zero regulation error with using an infinite, though countable, dimensional internal model.

\section{REFERENCES}

[1] D. Astolfi, A. Isidori, L. Marconi and L. Praly, "Nonlinear Output Regulation by Post-processing Internal Model for Multi-Input Multi-Output

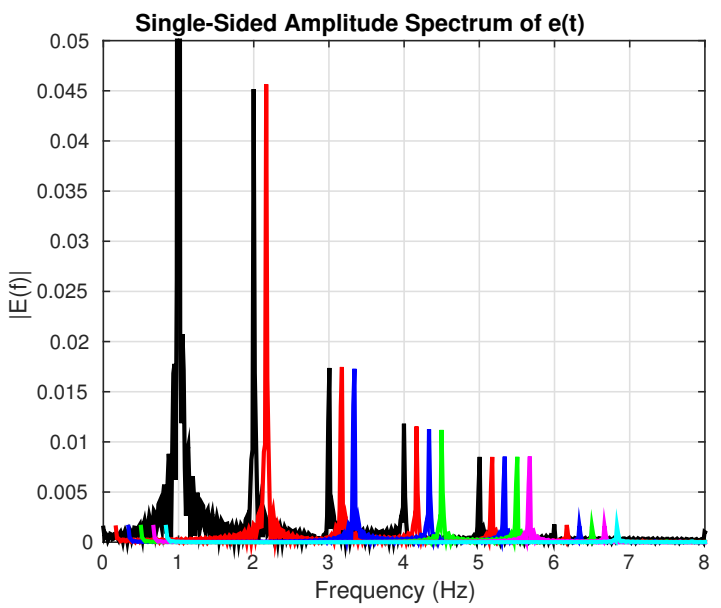

Fig. 3: Frequency spectrum of the output $e(t)$ in absence of the internal model (black) and in presence of the internal model with $L=1$ (red), $L=2$ (blue), $L=3$ (green), $L=4$ (magenta) and $L=5$ (cyan). Note that for graphical reason the frequencies of the signals have been right-shifted to avoid overlapping.

Systems", 9th IFAC Symposium on on Nonlinear Control Systems, pp: 295-300, 2013.

[2] C. I. Byrnes, F. Delli Priscoli, A. Isidori, "Output Regulation of Uncertain Nonlinear Systems”, Birkhäuser, 1997.

[3] C. I. Byrnes and A. Isidori, "Nonlinear Internal Models for Output Regulation", IEEE Trans. Automatic Control, Vol. 49, No. 12, 2004.

[4] F. Celani, "Certainty equivalence in nonlinear output regulation with unmeasured error", International Journal of Control, Vol. 83, No. 11, 2195-2203, 2010.

[5] F. Delli Priscoli, "Robust tracking for polynomial plants", Proc. of 2nd European Control Conf. pp. 369-373, 1993.

[6] F. Delli Priscoli, "Sufficient conditions for robust tracking in nonlinear systems", International Journal of Control, Vol. 67, No. 5, pp. 825-836, 1997.

[7] B. A. Francis and W. M. Wonham, "The Internal Model Principle of Control Theory", Automatica Vol. 12, pp. 457-465, 1976.

[8] J. Huang, C. F. Lin, "Internal model principle and robust control of nonlinear systems", 32th IEEE Conference on Decision and Control, pp. 1501-1506, 1993.

[9] A. Isidori, "A Tool for Semiglobal Stabilization of Uncertain NonMinimum-Phase Nonlinear Systems via Output Feedback", IEEE Transaction on Automatic Control, Vol 45, No. 10, pp. 1817-1827, 2000.

[10] A. Isidori and C. I. Byrnes, "Output Regulation of Nonlinear Systems", IEEE Transactions on Automatic Control Vol. 35, No. 2., pp. 131-140, 1990.

[11] A. Isidori and L. Marconi, "A Zero-Dynamics Re-Design Approach to Problems of Nonlinear Output Regulation", Dynamics of Continuous Discrete and Impulsive Systems, Series A Mathematical Analysis, 17, pp. 809-827, 2010.

[12] A. Isidori and L. Marconi, "Shifting the internal model from control input to controlled output in nonlinear output regulation", 51th IEEE Conference on Decision and Control, pp: 4900-4905, 2012.

[13] H. K. Khalil, "Robustness servomechanism output feedback controllers for a class of feedback linearizable systems", oral presentation at Workshop on nonlinear control systems, St. Louis, Missouri, 1992.

[14] L. Marconi, L. Praly, A. Isidori, Output Stabilization via Nonlinear Luenberger Observers, SIAM J. Contr. and Optimiz. 45, No. 6, pp. 22772298, 2007.

[15] F. Mazenc and L. Praly, "Adding integrations, saturated controls, and stabilization for feedforward systems", IEEE Transactions on Automatic Control, Vol. 41, No. 11, pp. 1559-1578, 1996.

[16] S. Nazrulla, H. K. Khalil, "Output Regulation of Non-Minimum Phase Nonlinear Systems Using Extended High-Gain Observers", 18th IFAC World Congress, pp. 1386-1391, 2011.

[17] F. Poulain and L. Praly, "Robust asymptotic stabilization of nonlinear systems by state feedback", 8th IFAC Symposium on Nonlinear Control Systems, pp. 653-658, 2010.

[18] T. Yoshizawa, "Stability Theory by Liapunov's Second Method", Publications of the Mathematical Society of Japan, Japan, 1966. 